\title{
PROGRESS ON THE CONTINUOUS OPTICAL FAST CVD SYSTEM TO GROW SILICON RIBBONS FOR SOLAR CELLS VIA SDS PROCESS
}

\author{
Augusto A. ${ }^{1,2}$, Serra J.M. ${ }^{1,2}$, and Vallêra A.M. ${ }^{1,2}$ \\ ${ }^{1}$ Faculty of Science University of Lisbon, SESUL, Lisbon, Portugal \\ ${ }^{2}$ MIT-Portugal Program, Portugal/USA
}

\begin{abstract}
In this paper we present the progress on the development of the Continuous Optical Fast CVD (COFCVD) system based on the Silicon on Dust Substrate (SDS) process. The SDS process is a ribbon technology [1], where ribbons are grown on top of a silicon dust substrate, directly from a gas precursor, silane. Besides the advantages of avoiding kerf losses, the growth of silicon ribbons directly from a gaseous feedstock has the benefit of bypassing also unnecessary crystallization processes such as the Siemens process (see Figure 1).
\end{abstract}

\section{INTRODUCTION}

Silicon wafers in PV modules account for almost half of its cost. The ingot wafering process originates kerf losses and significantly contributes to increased costs. These losses in the ribbon technologies are negligible since no sawing processes are involved. Growing ribbons directly from gaseous feedstock has as additional advantage to prevent unnecessary crystallization processes (e.g. Siemens) that are costly in terms of energy and time.

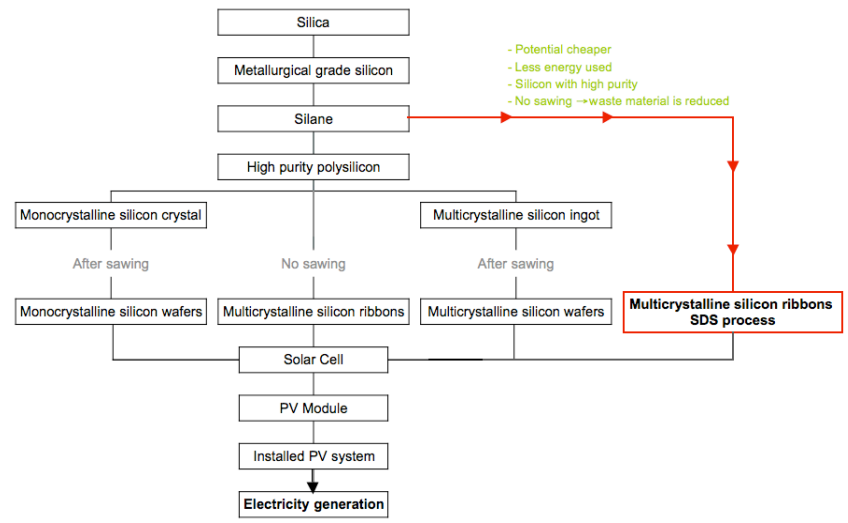

Figure 1 Basic producing steps of a PV system based on c-Si materials

\section{RIBBON GROWTH}

Epitaxial crystalline silicon film growths rates are typically low, a requirement for good crystalline quality. For instance, using silane in the low temperature regime (< $900^{\circ} \mathrm{C}$ ), the growth rates are typically $10^{\prime}$ s of $\AA / \min$ [2].

In the SDS process we addressed this problem by decoupling the deposition from the crystallization step, seeking for higher deposition rates - up to $20 \mathrm{micron} / \mathrm{min}$. Thus the SDS is a two-step process: first a self-standing pre-ribbon is grown by COFCVD on top of silicon dust (sacrificial layer) using silane and than in a second step, the pre-ribbon is crystallized by a floating zone method, acquiring a proper (multi)crystalline structure for solar cells use. The pre-ribbon term is applied instead of just ribbon, because the grown ribbon has a porous and microcrystalline structure, as result of a fast deposition process at atmospheric pressure and low temperature.

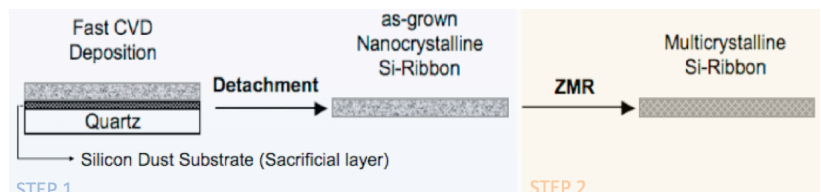

Figure 2 Diagram of SDS process

The pre-ribbon is crystallized by a floating molten zone technique, known as zone melting recrystallization (ZMR), increasing its crystal quality while avoiding impurity contamination. The ZMR step is performed using an inhouse-developed furnace composed of two elliptical mirrors that concentrate, on the pre-ribbon, the radiation of two 1000W halogen lamps [3]. The doping is achieved by a recently developed technique [4], where boric acid is sprayed over the pre-ribbon surface prior to the crystallization step.

As a process the SDS has the following advantages: (i) no substrate (therefore no cost and no contamination); (ii) low thermal budget (ambient pressure, low temperature CVD); (iii) high quality and self-standing crystalline silicon sheet (float zone crystallization, no contact with foreign materials)

\section{CVD SETUP}

The COFCVD system idea comes from the need to upgrade the previous CVD reactor in order to overcome some fundamental issues, such as: increase the preribbons deposition homogeneity and evolve from a batch system to a continuous system ideal for inline production. The homogeneity is obtained in part due to the continuous nature of the reactor, where the substrate holder moves along the reactor, forcing all regions of the substrate to experience the same deposition conditions along the displacement direction. Regarding the homogeneity along the transversal direction, an apparatus of elliptical mirrors is used to ensure a uniform radiation distribution in this direction. 


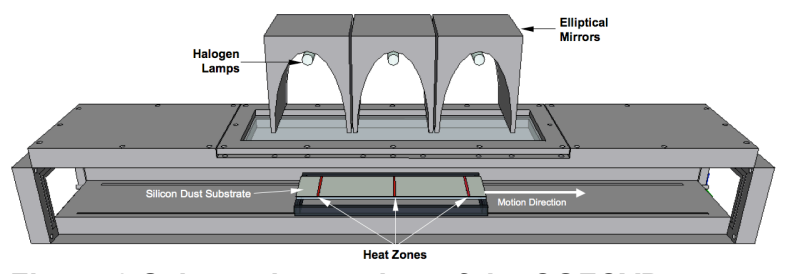

Figure 3 Schematic cut view of the COFCVD reactor

The silicon dust substrate is itself obtained from thermal dissociation of silane (Equation 1) and placed on top of a quartz holder plate that moves inside the reactor at a constant speed. The pre-ribbon grows on top of the silicon dust substrate also by thermal dissociation of silane (Figure 3).

$$
\mathrm{SiH}_{4}(g) \rightarrow \mathrm{Si}(s)+2 \mathrm{H}_{2}(g)
$$

As result a more homogenous deposition is obtained since all regions of the sample experience the same deposition conditions along the reactor. The thermal energy for the deposition comes from the radiation of three 1000W halogen lamps (not used at full power), placed on top of a reactor window. Each lamp has attached an elliptic mirror that focuses the radiation on the silicon dust substrate. As a result, three heating regions are defined (the red stripes in Figure 3). These heating regions origin well confined convection cells inside the reactor [5] that boost the deposition rate and the silane conversion to silicon.

\section{RESULTS}

So far it was possible to grow 6 to $10 \mathrm{~mm}$ of pre-ribbon per minute at temperatures below $800{ }^{\circ} \mathrm{C}$. The growth is strongly related with the substrate holder speed.

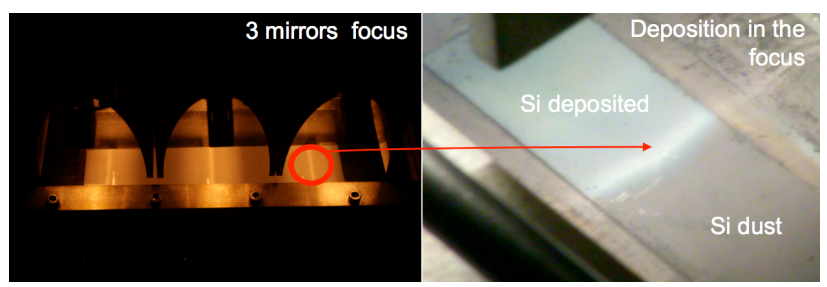

Figure 4 (Left) Focus of the three elliptical mirrors. (Right) Deposition of silicon while the substrate is passing (from right to left) by the focus of the first heating zone.

In order to access the deposition conditions of the reactor, the radiation distribution profiles were studied. Figure 5, shows the measured radiation profile along the substrate displacement direction, while the substrate passes through the three heating zones. As expected there is a very sharp and symmetric peak around the heating zones focus.

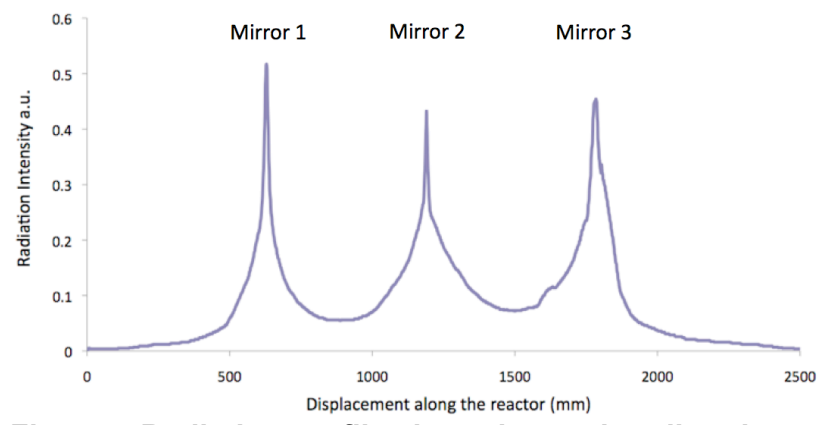

Figure 5 Radiation profile along the motion direction

Due to the elliptical geometry of the mirrors and the tubular shape of the lamps filament, the focus is a well defined line perpendicular to the displacement direction (red stripes in Figures 3 and 4) with 1-2 mm width. A homogeneous deposition requires a fairly steady radiation along these focus lines. The measurements (Figure 6) shows that the radiation profile in these focus lines is symmetric, but non-homogeneous as we go to the substrate borders. This issue is being addressed in the reactor right now.

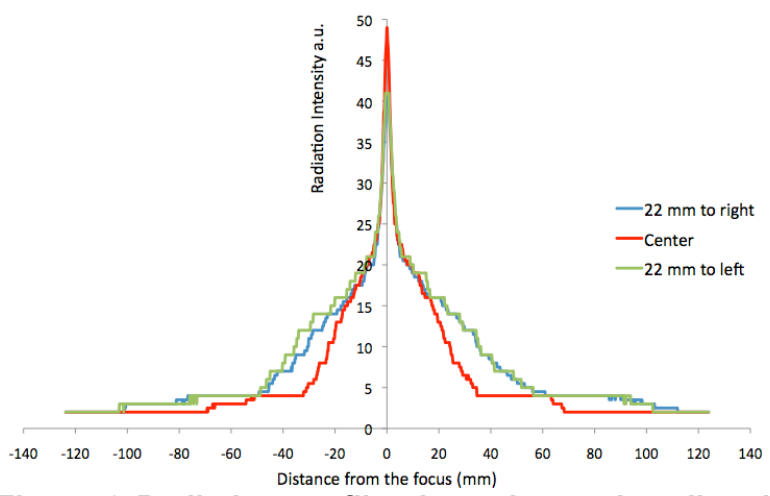

Figure 6 Radiation profile along the motion direction for different positions in the focus line

The obtained pre-ribbons are mechanically self-standing having an amorphous structure, as can be seen in Figure 7. Its thicknesses are round the $300 \mu \mathrm{m}$, however it is expected a thickness decrease after the crystallization step. 


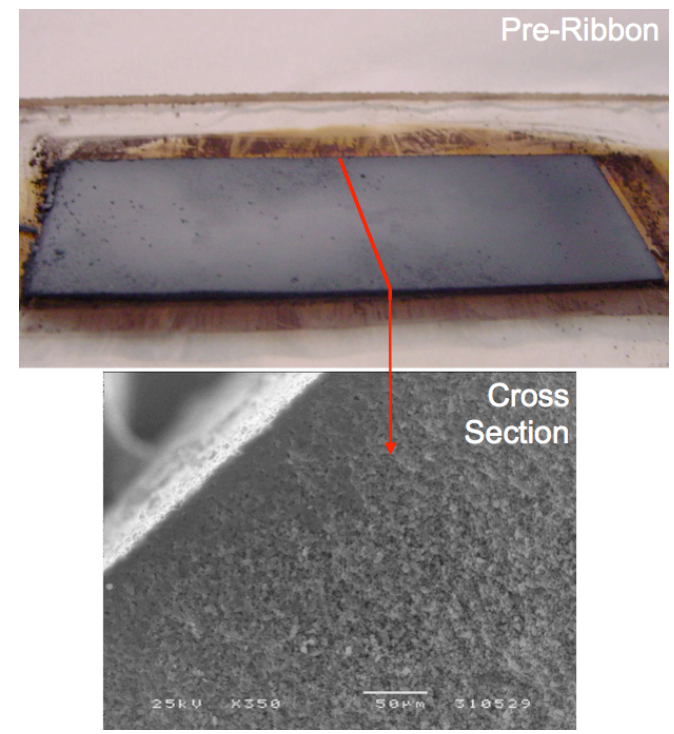

Figure 7 (Top) Pre-ribbon after deposition. SEM image of pre-ribbon cross section

\section{CONCLUSIONS}

Self-supported pre-ribbons were obtained using a continuous CVD deposition process with 6 to $10 \mathrm{~mm} / \mathrm{min}$ ribbon growth rate. The radiation distribution along the reactor as predicted [5] shows 3 sharp peaks at the mirrors focus. In the transversal direction some engineering as yet to be applied to guarantee the radiation homogeneity along this direction. The next step will be the pre-ribbon ZMR crystallization step to make solar cells with this material.

\section{ACKNOWLEDGEMENTS}

This work has been supported by FCT grant: SFRH/ BD/ 39385/ 2007; FCT project: PTDC/ENR/67616/2006. The authors also acknowledge the financial support of LusoAmerican Development Foundation.

\section{REFERENCES}

[1] Serra J M et al, The SDS path to make solar cells directly from a gaseous feedstock, Semicond. Sci. Technol. 24, 045002; doi:10.1088/0268-1242/24/4/045002

[2] Michael A. Todd et al, Low temperature, high growth rate epitaxial silicon and silicon germanium alloy films, Applied Surface Science 224 (2004) 41-45

[3] Pinto C.R., Serra J. M., Brito M. C., Gamboa R., Maia Alves J., Vallêra A. M.; "Zone melting recrystallization of self supported silicon ribbons obtained by fast cvd from silane"; Proceedings of the 21st EPVSEC, Dresden 2006.
[4] Silva J. A., Brito M. C., Costa I, Maia Alves J., Serra J. M. and Vallêra A. M. 2007; Sol. Energy Mater. Sol. Cells 91, 1948-53.

[5] Pera D., Augusto A., Maia Alves J, Brito M.C., Serra J.M. and Vallêra A.M; "Inline Fast CVD System for continuous production of Silicon ribbons for solar cells by the SDS process"; Proceedings of the $34^{\text {th }}$ IEEE PVSC, Philadelphia, 2009. 